Onkologie 1989;12(suppl. 1):1

\title{
Inhalt, Vol. 12, Supplement 1, 1989
}

Impressum Editorial .

Contents

Imprint . Editorial

Hinweise für Autoren.

Instructions to Authors

Schalk, K. P.; Rütteńans, H.; Kaltwasser, J. P.; Said l-Hadj, Staffenberger, L. (Frankfurt) Tumorerkennung im Serum durch NMR-Spektrometrie. Eine kritische Überprüfung des sogenannten Fossel-Tests 5

Gussetis, E. S.; Ebener, U.; Wehner, S.; Komhuber, B.

(Frankfurt)

Immunologische Identifizierung von durch herkömmliche zyto-

histologische Untersuchungen an Knochenmarksproben nicht

erkennbare Neuroblastomzellen

9

Bares, R.; Faß, J.; Weíller, G.; Walter, S.; Kleinhans, E.;

Truong, S.; Bull, U.; Schumpelick, V. (Aachen)

Klinische Bedeutung der Immunszintigraphie für Diagnose und

Therapie gastrointestinaler Malignome 13

Halting, Th,; Steinbächer, M.; Kretzschmar, U.; Georgi, P.; Schlag, P. (Heidelberg)

Verbessert die Immunszintigraphie die Frühdiagnostik des meta-

stasierten oder rezidivierten Karzinoms? 20

Baum, R. P.; Lorenz, M.; Hertel, A.; Baew-Christow, Th.; Schwarz, A.; Hör, G. (Frankfurt)

Erfolgreiche immunszintigraphische Tumordetektion mit Tech-netium-99m-markierten

monoklonalen Anti-CEA-Antikörpern . 26

Pfreundschuh, M.; Da Costa, L.; Diehl, V. (Köln),

Carde, P.; Manil, L.; Lumbroso, J.-D.; Caillou, B.; Boudet, F.;

Ricard, M.; Hay at, M.; Parmentíer, C. (Villjuif/Frankreich),

Saccavini, J.-C. (Gif-sur-Yvette/Frankreich)

In-vivo-Imaging von Hodgkin-Lymphomen mit monoklonalen

Antikörpern 30

Gückel, F.; Döhner, H.; Knauf, W.; Ho, A. D.; Semmler, W.;

van Kaick, G. (Heidelberg)

MR-tomographischer Nachweis von Knochenmarkinfiltrationen

bei malignen Lymphomen 34

Ruhlmann, J.; Bockisch, A.; Dewes, W.; Loos, U.; Hotze, A.; Biersach, H.-J. (Bonn)

Knochenmarkszintigraphie und Magnetresonanztomographie

beím Plasmozytom 38 
Jeske, J.; Herholz, K; Heindel, W.; Heiß, W. D. (Köln)

Stofíwechseluntersuchungen an Gliomen mit der Positronen-

Emissions-Tomographie und der Phosphor-31-MR-Spektrosko-

pie in Diagnostik und Therapieplanung $\quad 42$

Semmler, W.; Bachert-Baumann, P.; Gückel, F.;

Gademann, G.; 'Label, H.-J.; van Kaick, G. (Heidelberg)

Nicht-invasive Tumortherapieverlaufskontrolle nach Chemothe-

rapie mit Hilfe der 31P-MR-Spektroskopie an einem Ganzkör-

pertomographen $\quad 46$

Port, R. E.; Strauss, L. G.; Cloríus, J. H. (Heidelberg)

Positronen-Emissions-Tomographie nach Kurzinfusion von

5-[18F]Uracil: Lineares Modell für die Kinetik der 18F-Radioakti-

vität in Tumoren 51

Schalk, K. P.; Rütteńans, H.; Kaltwasser, J. P.; Said 1-Hadj,

Staff enberger, L. (Frankfurt)

Detection of Cancer by NMR-Spectrometry. Critical Evaluation

of the So-Called Fossel Test 5

Gussetis, E. S.; Ebener, U.; Wehner, S.; Komhuber, B. (Frankfurt)

Immunological Detection of Neuroblastoma Cells in Bone Mar

row Samples not Recognizable by Conventional Cytohistological

Investigations 9

Bares, R.; Faß, J.; Waller, G.; Wolter, S.; Kleinhans, E.;

Truong, S.; Bull, U.; Schumpelick, V. (Aachen)

Clinical Impact of Immunoscintigraphy of Gastrointestinal

Malignomas 13

Halting, Th.; Steinbächer, M.; Kretzschmar, U.; Georgi, P.; Schlag, P. (Heidelberg)

Can Immunoscintigraphy Improve the Early Diagnosis of Metas-

tasized or Recurrent Carcinoma? 20

Baum, R. P.; Lorenz, M,; Hertel, A.; Baew-Christow, Th.; Schwarz, A.; Hör, G. (Frankfurt)

Successful Immunoscintigraphic Tumor Detection with Tc-99m

Labeled Monoclonal Anti-CEA Antibodies 26

Pfreundschuh, M.; Da Costa, L.; Diehl, V. (Köln),

Carde, P.; Manil, L.; Lumbroso, J.-D.; Caillou, B.; Boudet, F.;

Ricard, M.; Hay at, M.; Parmentier, C. (Villjuif/France),

Saccavini, J.-C. (Gif-sur-Yvette/France)

In vivo Imaging of Hodgkin's Lymphomas with Monoclonal

Antibodies 30

Gückel, F.; Döhner, H.; Knauf, W.; Ho, A. D.; Semmler, W.; van Kaick, G. (Heidelberg)

MRT-Detection of Bone Marrow Involvement by Malignant

Lymphoma 34

Ruhlmann, J.; Bockisch, A.; Dewes, W,; Loos, U.; Hotze, A.; Biersach, H.-J. (Bonn)

Bone Marrow Scintigraphy and Magnetic Resonance Tomo

graphy in Plasmocytoma 38

Jeske, J.; Herholz, K; Heindel, W.; Heiß, W. D. (Köln)

Measurement of Metabolism in Gliomas by Positron Emission 
Tomography and Phosphorus-31-MR-Spectroscopy in Diagnosis

and Therapy Management

42

Semmler, W.; Bachert-Baumann, P.; Gückel, F.;

Gademann, G,; Zabel, H.-J.; van Kaick, G. (Heidelberg)

Non-Invasive Tumor Therapy Response to Chemotherapy by

Means of in vivo 31P MR Spectroscopy Using a Whole-Body

MR-System 46

Port, R. E.; Strauss, L. G.; Cloríus, J. H. (Heidelberg)

A Linear Model to Describe the Kinetics of $18 \mathrm{~F}$ in Tumors

Following Brief Infusion of S-p, $3 / 8$ JUracil, When Radioactivity is

Monitored by Positron Emission Tomography 51

Alle publizierten Arbeiten wurden anläßlich des 19. Deutschen Krebskongresses 1988 in Frankfurt, vorgetragen. Zusammengestellt von: M. Pfreundschuh, Köln und G. van Kaick, Heidelberg

All published lectures were presented at the 19th German Cancer Congress 1988 in Frankfurt. Compiled by: M. Pfreundschuh, Köln and G. van Kaick, Heidelberg 1989;012:1 